\title{
Guest Editorial Sensor Informatics for Managing Mental Health
}

$\mathbf{M}$ ENTAL, neurological, and substance-use (MNS) disorders such as depression, psychosis, bipolar disorders, epilepsy, developmental, and behavioral disorders in children and adolescents, dementia, Parkinson's disease, alcohol use disorders, drug use disorders, and suicidal behavior have high prevalence worldwide. They are major contributors to morbidity and premature mortality and currently constitute $13 \%$ of the global burden of disease, surpassing both cardiovascular disease and cancer [1]. Besides disease symptoms and related disabilities/consequences, MNS disorders are a source of significant economic burden, including direct costs (e.g., medical services and prescription drug costs), suicide-related costs, and workplace costs (e.g., missed days of work, and reduced productivity while at work).

Historically, mental and substance use disorders were not a global health priority, especially when compared with other severe somatic diseases. Nowadays, although in many countries (especially the developing ones) financial resourcing is not commensurate with the actual disease burden, healthcare services for mental disorders are typically not neglected. To this extent, it is worthwhile mentioning the World Health Organization's Comprehensive Mental Health Action Plan 2013-2020, which aims to promote an improvement in living and working environments to reduce the incidence of mental disorders, to provide appropriate psychiatric care to individual countries and cultures, and to recognize the rights of people with mental health issues.

Nowadays, in neurology, objective diagnostic tools such as brain imaging play a central role in daily clinical care, particularly for disease diagnosis. However, many questions are still open and unanswered for many neurological disorders, such as how to early diagnose Parkinson's disease and how to predict seizure onset in epilepsy.

In psychiatry and, more in general, in many mental and substance use disorders, objective tools are still underdeveloped or not yet clinically applicable since the pathophysiology related to these disorders is still not well understood. As a matter of fact, mental and substance use disorders are usually diagnosed relying on thresholds and criteria described in clinical guidelines such as the Diagnostic and Statistical Manual of Mental Disorders or the International Classification of Diseases. Clearly, a diagnosis exclusively based on clinician observation and patient's subjective description can bring to biased interpretation and inconsistency. These challenges related to diagnosis, monitoring, treatment, and prevention of MSN disorders surely reflect that synergistic and multidisciplinary research in this field is strongly needed.

Digital Object Identifier 10.1109/JBHI.2016.2565758
To this end, novel avenues of biomedical and health informatics technologies need to focus on innovative sensing and processing platforms for effectively capturing information during daily activities or sleep, during specific tasks, at home, and in clinics for the assessment of the subjects' psychophysiological state including affective, stress, mood, and behavioral states. Research involving such computational models of pathophysiology and related signal/data processing surely have the potential to guide the future delivery of treatment tailored to individual needs, improving the lives of people affected by MNS disorders and to support clinicians dealing with MNS diagnoses and treatments. Intersubject variability, in fact, is very high in MNS-related pathologies.

This special issue "Sensor Informatics for Managing Mental Health" provides novel insights on advances in detection, sensing, analysis, and modeling of central and/or autonomic correlates useful in psychophysiological states assessment. Specifically, Akay et al. [2] developed a weighted network model to represent user activity on social health networks. This approach could be exploited deriving side-effects and consumer sentiment on, e.g., drugs for depression treatment. Saha et al. [3] developed a framework for classifying online mental health related communities with an interest in depression. This allows for providing a significant resource for studies on textual signs of psychological health problems. Tai et al. [4] built a feeling distinguisher system for detecting a person's intention and intensity of feelings through the analysis of his/her online posts. This can have a strong impact also on recognizing potential mental illnesses. Zhang et al. [5] described a method for a patientspecific epileptic seizure onset and termination detection, and related on-chip hardware implementation. Hernando et al. [6] investigated how respiratory rate and heart rate variability can be profitably used as a stress marker in young adults; thus, objectifying current assessment based on questionnaires scores. Ciulli et al. [7] developed a computational approach to predict the loss in executive functions in patients with mild cognitive impairment and cerebral small vessel disease. This also allows for the assessment of specific brain substrates of these impairments. Valenza et al. [8] proposed a signal processing method, based on heart rate variability analysis, to objectively assess and predict mood changes in bipolar disorder from long-term cardiovascular monitoring.

These selected studies point out current research directions in advancing our understanding of diagnosis and treatment of MNS disorders. First, it is clear that a significant effort is being focused on social (health) networks, whose large amount of data surely calls for the development of ad hoc analysis, preferably with capabilities of inference at a single-user level and realtime detection of specific (may be clinical) features. Second, it 
is important to highlight that major aims in sensor informatics for MSN are related to new devices, methods, and computational models employed to objectify current subjective clinical assessments. Ideally, clinical assessments made through scores and structured interviews should be replaced with quantifiers from biological/physiological data, whose monitoring and processing should be performed in a noninvasive, comfortable, and wearable fashion, when possible. Third, major breakthroughs of technologies for MNS disorders are likely related to the prediction of the onset of severe events, especially in chronic and degenerative aberrations.

We believe that future endeavors of sensor informatics for managing mental health, aiming to improve diagnose and treatment of MNS, should account for recent avenues in genomics and neuroscience, along with the investigation of specific sociocultural and environmental contexts. This would also help achieving the goal of patient-specific modeling, allowing us to guide the delivery of treatment tailored to individual needs.

Importantly, it is well known that mental health strongly affects "body" health. In fact, bidirectional brain-body pathways can be thought of as the substrate mediating the relationship between psychological and social factors and physical health. As a significant example, brain disorders may lead to cardiovascular disorders and vice-versa.

A major limitation for this kind of multivariate analysis concerns the amount of data available: it is very difficult and time consuming, in fact, to obtain brain scans along with long-term monitoring of autonomic nervous systems signals, scores from psychological assessments, and genomics data from thousands of (homogeneous) individuals. In this view, the current scientific community, including clinical organizations, should deeply invest in online repositories for healthcare data, and sort of "unambiguous" inclusion/exclusion criteria for subjects/patients enrollment.

This editorial effort would not have been possible without the thorough and helpful effort of all the reviewers and the editorial team of the IEEE JOURNAL OF BIOMEDICAL AND HEALTH INFORMATICS. Our effort to bring together a coordinated international collaborative effort from leading technical experts resulted in a significant special issue focused on technologymental health interplay. Therefore, we expect that a growing recognition of the importance of developing technology for MNS-use disorders will make researchers and physicians of the future more aware of dealing with such complex systems, which in turn will provide them with a broader perspective when evaluating and treating patients.

\author{
GAETANo VALENZa Editor \\ University of Pisa, Pisa \\ Italy and Mass. General Hospital/ \\ Harvard Medical School \\ Boston, USA \\ g.valenza@ieee.org \\ VLADIMIR CARLI Editor \\ Karolinska Institutet \\ Stockholm, Sweden \\ vladimir.carli@ki.se \\ ANTONIO LANATA Editor \\ University of Pisa, Pisa, Italy \\ a.lanata@centropiaggio.unipi.it \\ WEI CHEN Editor \\ Fudan University, China \\ w_chen@fudan.edu.cn \\ ROOZBEH JAFARI Editor \\ Texas A\&M University, USA \\ rjafari@tamu.edu \\ ENZo Pasquale ScILINGo Editor \\ University of Pisa, Pisa, Italy \\ e.scilingo@ing.unipi.it

\section{REFERENCES}

[1] P. Y. Collins et al., "Grand challenges in global mental health," Nature, vol. 475, no. 7354, pp. 27-30, 2011.

[2] A. Akay, A. Dragomir, B.-E. Erlandsson, and M. Akay, "Assessing antidepressants using intelligent data monitoring and mining of online fora," IEEE J. Biomed. Health Informat., 2016, to be published.

[3] B. Saha, T. Nguyen, D. Phung, and S. Venkatesh, "A framework for classifying online mental health related communities with an interest in depression," IEEE J. Biomed. Health Informat., 2016, to be published.

[4] C-H. Tai, Z.-H. Tan, and Y.-S Chang, "Systematical approach for detecting the intention and intensity of feelings on social network," IEEE J. Biomed. Health Informat., 2016, to be published.

[5] C. Zhang, M. Altaf, and J. Yoo, "Design and implementation of an onchip patient-specific closed-loop seizure onset and termination detection system," IEEE J. Biomed. Health Informat., 2016, to be published.

[6] A. Hernando et al., "Inclusion of respiratory frequency information in heart rate variability analysis for stress assessment," IEEE J. Biomed. Health Informat., 2016, to be published.

[7] S. Ciulli et al., "Prediction of impaired performance in trail making test in MCI patients with small vessel disease using DTI data," IEEE J. Biomed. Health Informat., 2016, to be published.

[8] G. Valenza et al., "Predicting mood changes in bipolar disorder through heartbeat nonlinear dynamics," IEEE J. Biomed. Health Informat., 2016, to be published. 